\title{
New Treatment for Psoriasis: Therapeutic Efficiency in Patients and Antiinflammatory Effect in Vitro
}

\author{
Ignacio Umbert ${ }^{1,3 *}$, Leonardo Márquez-Kisinousky ${ }^{4,5}$, Consol Farrera ${ }^{2}$, Jordi Valls ${ }^{1}$, Annabel Valledor ${ }^{2}$ and \\ Laura Fernández-Fernández ${ }^{4}$ \\ ${ }^{1}$ Barcelona Skin Genomic SL, Barcelona, Spain \\ ${ }^{2}$ Department of Cell Biology, Physiology and Immunology, Faculty of Biology, University of Barcelona, Spain \\ ${ }^{3}$ Instituto de Dermatología Ignacio Umbert Plaza Manuel Corachán 4, Barcelona, Spain \\ ${ }^{4}$ Medical Science Consulting, Gijón, Spain \\ ${ }^{5}$ Department of Brain Ischemia and Neurodegeneration, Barcelona Institute for Biomedical Research (IIBB) -Higher Council for Scientific Research \\ (CSIC), Barcelona, Spain
}

Submission: May 27, 2020; Published: June 09, 2020

*Corresponding author: Ignacio Umbert, Barcelona Skin Genomic SL and instituto de Dermatología Ignacio Umbert Plaza Manuel Corachán 4, Barcelona, Spain

Abstract

Background: Psoriasis is a worldwide chronic inflammatory disease that is increasingly being considered as a systemic disorder. Antioxidant and anti-inflammatory molecules have been revealed as potential adjuvants for traditional therapies.

Objectives: To evaluate the antiinflammatory action of selected antioxidant molecules and their beneficial effect as combination therapy in psoriatic patients.

Materials \& Methods: Thirty-five patients with mild (five), moderate (nine) and severe (twenty one) psoriasis were selected and treated with EP2311454 treatment. PASI, BSA and DLQI assessed the severity of psoriasis and the impact on quality of life. The antiinflammatory effect was tested in IFN- $\gamma$ stimulated Raw264.7 macrophages that express human PPARG.

Results: The combined use in vitro of several EP2311454 components exerts a synergistic inhibitory effect on the expression of the proinflammatory mediators Cox2, Nos2 and Cxcl9. Clinically, more than $80 \%$ reduction in DLQI levels is observed after treatment, and $100 \%$ of severe patients achieved PASI 75 and significant reduction of BSA levels after 15 days of treatment.

Conclusion: EP2311454 treatment, composed by antioxidant molecules, proved to be antiinflammatory in vitro and showed efficacy in patients with mild to severe psoriasis, as demonstrated by a significant decrease in PASI, BSA and DLQI levels.

Keywords: Antiinflammatory; Antioxidant; Psoriasis Treatment

\section{Introduction}

Psoriasis is a chronic inflammatory disease with predominant skin and joint involvement and a worldwide distribution affecting both sexes, of any age, but more frequently in the $3^{\text {rd }}$ and $4^{\text {th }}$ decades of life [1]. The disease is characterized by disfiguring scaling and erythematous plaques. The severity of psoriasis is defined by the clinical form, the proportion of body surface area affected and the severity of the symptoms. Psoriatic plaques may be painful and often severely pruritic, causing a deterioration in the quality of life [2]. Medical therapy for psoriasis has classically included topical and systemic treatments such as phototherapy, retinoids, methotrexate, and cyclosporine [3]. In the last few years, biologic treatment has become the established therapeutic standard for significant improvement of the quality of life of patients with moderate to severe psoriasis [4]. The involvement of oxidative stress in the pathogenesis of psoriasis has been recently proposed. Oxidative stress is an imbalance between 
oxidants and antioxidants in favour of the oxidants, leading to a disruption of redox signalling and promoting molecular damage [5]. Different studies have associated the antioxidant properties of specific compounds with anti-inflammatory effects in psoriasis, suggesting a potential role for these compounds as therapeutic tools [6,7]. Using well characterized compounds, the "Ignacio Umbert Dermatology Institute" (IUDI) has developed topical treatments that differ from the standards. The therapy used for psoriasis (European patent number EP2311454) is a combination of well-established drugs and a set of compounds with antioxidant activity (indomethacin, ascorbyl palmitate, taurine, curcumin and resveratrol), whose anti-inflammatory effects were tested in vitro in this study.

\section{Methods}

\section{Cell culture}

Raw264.7 macrophages (American Type Culture Collection, Manassas, VA, USA) were used for the in vitro analysis of the anti-inflammatory activity of specific compounds. Raw264.7 cells were stably transfected with a mammalian expression vector encoding human peroxisome proliferator-activated receptorgamma (PPARG). Cell transfection was performed using Superfect reagent (Qiagen, Valencia, CA, USA) following the manufacturer's recommendations. Two days after transfection, stable transfectants were selected by culturing the cells for two weeks in DMEM (Biowest, Nuaille, France) supplemented with 10\% heatinactivated fetal bovine serum (FBS) (Biosera, Boussens, France) and geneticin (Thermo Fisher Scientific, Rockford, IL, USA). A clone of cells was selected and the expression of human PPARG was verified by both quantitative real time-PCR and by western blotting using rabbit polyclonal antibodies against hPPARG (Cell Signaling, Danvers, MA, USA).

Treatment: Interferon-gamma (IFN- $\gamma$ ) was obtained from Thermo Fisher Scientific (Rockford, IL, USA). Indomethacin, ascorbyl palmitate, taurine, curcumin and resveratrol are the main components of EP2311454 treatment (EP2311454 patent). Ascorbic acid 6-palmitate was purchased from Abcam (Cambridge, UK) and the rest of compounds were obtained from Sigma-Aldrich (Saint Louis, MO, USA). All of them were dissolved according to the manufacturer's instructions. Treatment of Raw 264.7 cells with antioxidant compounds and/or IFN- $\gamma$ was performed in DMEM supplemented with $1 \%$ heat-inactivated FBS.

Determination of gene expression: Cells were washed twice in cold PBS and total RNA was extracted using Tri reagent (Sigma-Aldrich) as recommended by the manufacturer. For cDNA synthesis, $1 \mu \mathrm{g}$ of total RNA was subjected to reverse transcription using M-MLV Reverse transcriptase RNase H Minus, Point Mutant, oligo(dT)15 primer and PCR nucleotide mix (Promega, Fitchburg, WI, USA). Quantitative real time (rt)-PCR was performed using the Power SYBR Green Reagent Kit (Applied Biosystems, Foster City, CA) following the manufacturer's recommendations. The primers used in this study are shown in (Table 1). Annealing for all primers was performed at $60^{\circ} \mathrm{C}$ for $30 \mathrm{~s}$. Real-time monitoring of PCR amplification was performed using the ABI Prism 7900 Sequence Detection System (Applied Biosystems). Data were expressed as relative mRNA levels normalized to ribosomal L14 expression.

Table 1: Primers used for quantitative real time-PCR determinations.

\begin{tabular}{|c|c|c|}
\hline Gene name & Forward primer & Reverse Primer \\
\hline Cxc19 & 5'-GGAGTTCGAGGAACCCTAGTG-3' & 5'-GGGATTTGTAGTGGATCGTGC-3' \\
\hline L14 & 5'-TCCCAGGCTGTTAACGCGGT-3' & 5'-GCGCTGGCTGAATGCTCTG-3' \\
\hline Nos2 & 5'-GCCACCAACAATGGCAACA-3' & 5'-CGTACCGGATGAGCTGTGAATT-3' \\
\hline Cox2 & 5'-ATTCTTTGCCCAGCACTTCA-3' & 5'-GGGATACACCTCTCCACCAA-3' \\
\hline
\end{tabular}

\section{Human study}

Selection: A group of 35 patients, (5 mild, 9 moderate and 21 severe psoriasis) both males and females, previously diagnosed with psoriasis were included in the study. Patients were eligible to participate in the trial if they were at least 18 years of age and had been diagnosed with mild to severe psoriasis. Individuals with allergies or pregnant women were not included in the study. Patients provided written, informed consent.

Monitoring: For evaluation, a total of six visits were planned at $0,15,30,45,52$ and 60 days from the start of treatment. Photographs were taken and physical and dermatological examinations were performed.

Treatment: Patients were instructed to use $2 \mathrm{~g}$ of cream per application and perform three applications per day (every $8 \mathrm{~h}$ ).
The cream was placed first on the fingertips and then distributed homogeneously in small sub-portions on the affected area. Circular motions were performed until the cream was completely absorbed by the skin. In addition, $0.5 \mathrm{~g}$ curcumin per day was administered orally dissolved in soups.

Evaluation: The effectiveness of the therapy on disease severity was scored using two objective outcome measurements, namely Psoriasis Area Severity Index (PASI) and Body Surface Area (BSA). PASI was calculated for all patients. Each site (head, trunk, upper and lower limbs) was evaluated separately for erythema, infiltration and desquamation and the data were summed to calculate final PASI, as described previously ("Guideline on clinical investigation of medicinal products indicated for the treatment of psoriasis" European Medicines Agency Evaluation of Medicines for Human Use, 2014). Additionally, the quality of life was measured 
using the Dermatology Life Quality Index (DLQI). The following criteria was used to determine the severity of psoriasis: mild to moderate psoriasis: BSA $<10 \%$ or PASI $<10$; moderate psoriasis: $10 \%<$ BSA $>20 \%$ or $10 \leq$ PASI $\geq 20$; severe psoriasis: $\mathrm{BSA}>20 \%$ or PASI $>20$. The efficacy of treatment was evaluated through PASI 75 , which represents the percentage of patients achieving a $75 \%$ or more reduction in their PASI score from baseline.

\section{Statistical analysis}

Results are presented as mean \pm SEM. GraphPad Prism program (Prism 4.0, Graphpad Software Inc) and SPSS software (IBM) were used for statistical analysis of the human and in vitro studies, respectively. In Figure 3, a two-tailed Student t-test was used for paired comparisons. Differences were considered significant if the $\mathrm{p}$-value $<0.05$.

\section{Results}

\section{Clinical evaluation of EP2311454 in psoriasis patients}

Patients treated with EP2311454 have a positive response to treatment; examples of target-lesions improvement are shown in
Figure 1, in which the patients were recovered after 15 (Figure 1a) or 60 days (Figure $1 \mathrm{~b}$ ) of treatment. Quality of life before and after the treatment was assessed by determining the DLQI index and results indicate that EP2311454 improves the outcome. 95\% of patients treated show significant amelioration of the disease, with an average reduction of $80 \%$ reduction in DLQI levels after 60 days of treatment (Figure 1c). Besides DLQI, two other parameters, PASI and BSA, were evaluated at baseline and at different time points after the start of treatment (0, 15, 30, 45 and 60 days). PASI values decreased significantly, with clear effects already at day 15 of treatment (Figure 2a). When taking into account global improvement, $100 \%$ of patients, including patients with moderate to severe psoriasis, achieved at least $50 \%$ improvement in PASI (PASI 50) (Figure 2b) Furthermore, the proportions of patients who achieved PASI 75 was $80 \%$ in the mild to moderate group and $100 \%$ in the severe group of patients. Interestingly, $70 \%$ of EP2311454-treated severe patients reached PASI 90 (Figure 2b). On the other hand, significant reduction of BSA values compared to baseline were also observed in patients treated with EP2311454 (Figure 2c).
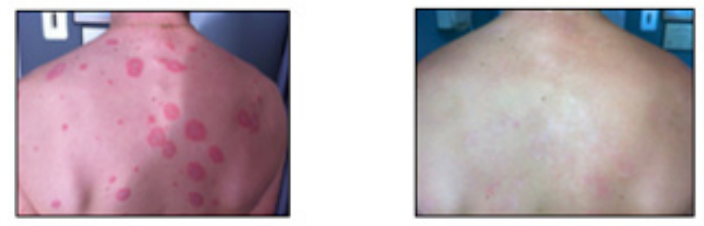

(A)
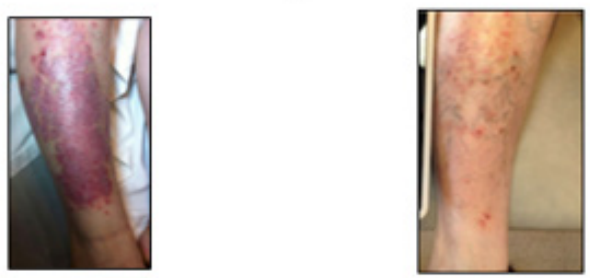

(B)



(C)

Figure 1: Progression of psoriatic lesions under EP2311454 treatment. a) Picture of the back of a patient with severe psoriasis before (left) and after (right) treatment. b) Picture of the arm of a moderate patient with psoriasis before (left) and after (right) treatment. c) DLQI levels pre and posttreatment, 60 days. Treatment reduces significantly DLQI levels $n=22{ }^{* * *} p<0.0001$ versus pretreatment (paired Student $t$-test). 


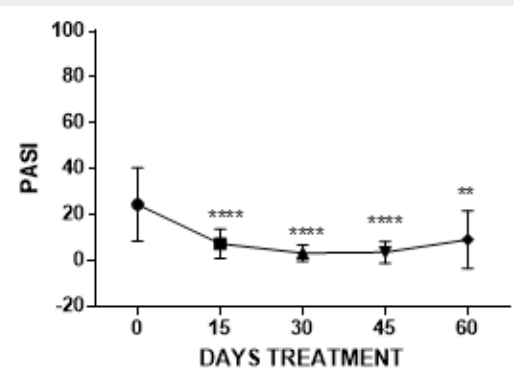

(A)

\begin{tabular}{|l|c|c|c|}
\hline & PASI 50 & PASI 75 & PASI 90 \\
\hline Mild & $100 \%$ & $80,0 \%$ & $40,0 \%$ \\
\hline Moderate & $100 \%$ & $77,8 \%$ & $44,4 \%$ \\
\hline Severe & $100 \%$ & $100,0 \%$ & $71,4 \%$ \\
\hline
\end{tabular}

(B)

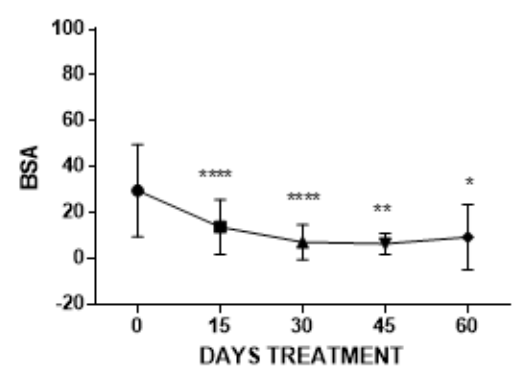

(C)

Figure 2: Therapeutic efficacy of treatment in patients with psoriaris. a) Psoriasis area severity index (PASI) quantifications during treatment. b) Percentage of patients with $50 \%$ reduction, $75 \%$ reduction or $90 \%$ reduction in the Psoriasis Area and Severity Index (PASI 50 , PASI 75 and PASI 90, respectively), compared with baseline, after treatment with EP2311454 The patients were divided in three groups according to the severity of psoriasis before treatment: mild $(n=5)$, moderate $(n=9)$ and severe $(n=21)$. c) Body Surface area (BSA) quantifications during treatment. Treatment reduces significantly PASI and BSA levels $(n=22){ }^{* * * *} p<0.0001$, ${ }^{* * *} p<0.001$ versus pre-treatment. Paired Student t-test.

\section{In vitro effects of EP2311454 macrophage activation by IFN- $\gamma$}

Raw264.7 macrophages expressing human PPAR- $\gamma$ were pretreated with different combinations of the antioxidant compounds in EP2311454 treatment and then stimulated with IFN- $\gamma$ for $6 \mathrm{~h}$ (Figure 3). Changes in gene expression were analyzed through quantitative rt-PCR (Figure 3a). The percentage of repression was calculated in order to compare the effectiveness of combinations of compounds (Figure 3b). In Figure 3b, positive values indicate repression (inhibition), whereas negative values indicate induction (increase) in comparison with the levels of expression in IFN- $\gamma$ treated cells. In these conditions, the combined use of curcumin and ascorbic acid 6-palmitate resulted in synergistic inhibitory effects on the expression of the enzymes cyclooxygenase 2 (Cox2) and nitric oxide synthase 2 (Nos2), and the chemokine Cxcl9, whereas the expression of tumor necrosis factor-alpha (Tnf $\alpha)$ and C-C motif chemokine ligand 2 (Ccl2/MCP-1) was unaffected (data not shown). Moreover, the use of indomethacin further enhanced the inhibitory effects of curcumin and ascorbic acid 6-palmitate on Nos2 and Cox2 expression.

\section{Discussion}

Given the limitations of current treatments for psoriatic diseases and the evidence that reactive oxygen species-mediated oxidative stress contributes to the pathogenesis of inflammatory skin disorders, including psoriasis [8,9], new approaches have been designed in the last few years based on the pharmacological use of antioxidant molecules, such as epigallocatechin-3-gallate [6] and curcumin [10]. Antioxidant compounds have demonstrated efficacy as therapeutical treatments also against other chronic inflammatory diseases [11]. Although the clinical application of antioxidants for skin inflammatory disorders is not widespread, we decided to test their efficacy in psoriasis using a combination of antioxidant compounds. In particular, EP2311454 combines compounds with both antioxidant and antiinflammatory activity and traditional drugs in order to ameliorate the symptoms of psoriasis. The main components in EP2311454 are resveratrol, taurine and ascorbyl-L-palmitate, previously defined as antioxidant compounds in humans, and indomethacin which is anti-inflammatory in low doses [12] 

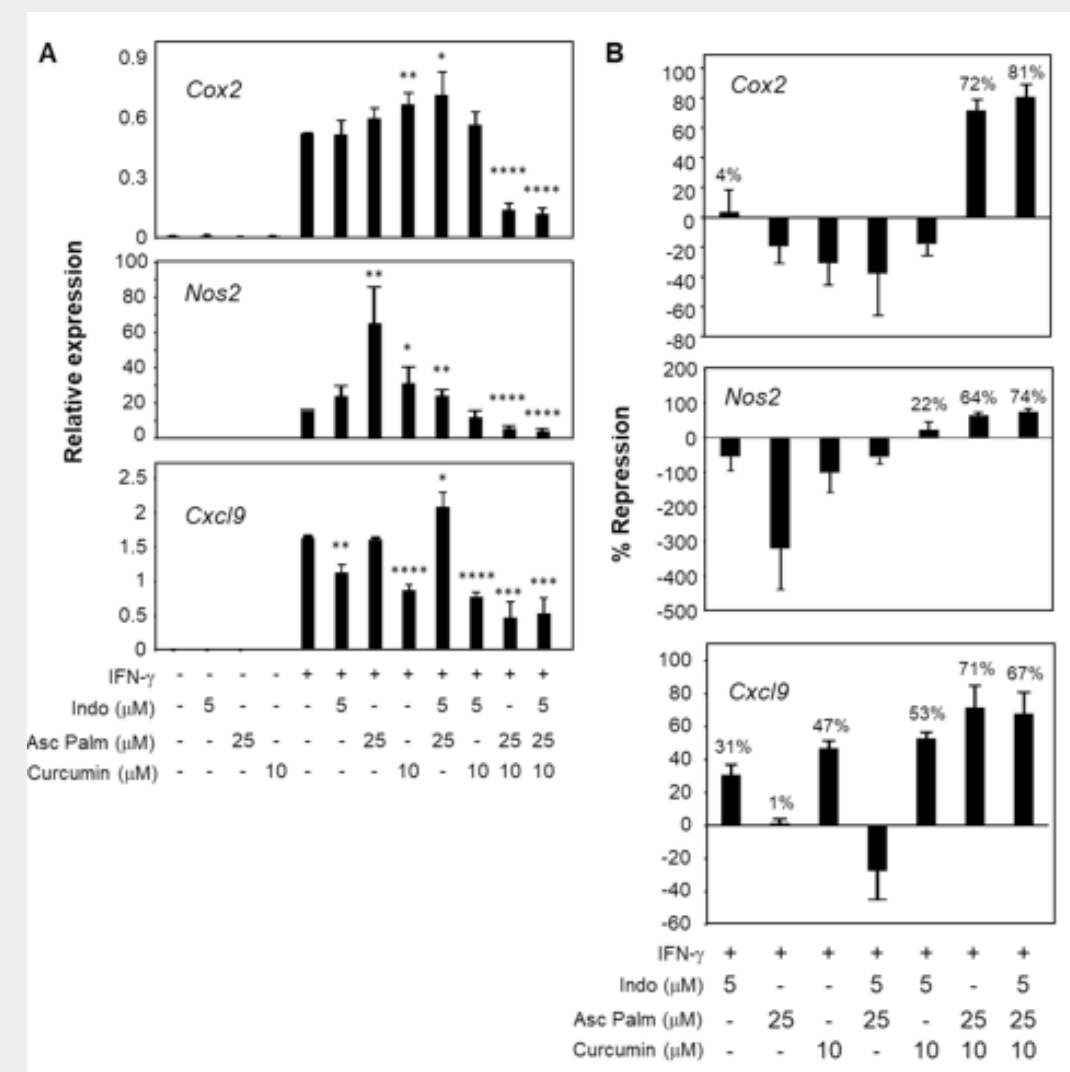

Figure 3: Effects of combined treatment with low doses of antioxidants. The cells were treated with IFN-y $(5 \mathrm{ng} / \mathrm{ml})$ in the presence or absence of the indicated antioxidants: indomethacin (Indo, $5 \mu \mathrm{M}$ ), ascorbic acid 6-palmitate (Asc. Palm, $25 \mu \mathrm{M})$, and/or curcumin (10 $\mu \mathrm{M}$ ). mRNA expression levels of several genes involved in the macrophage response to IFN- $y$ are represented a) The graphics show normalized values of gene expression relative to ribosomal L14 expression levels obtained from three to six independent experiments (depending on the gene studied). ${ }^{* * *} p<0.0001,{ }^{* * *} p<0.001,{ }^{* *} p<0.01,{ }^{*} p<0.05$ versus IFN-y. Two-tailed Student t-test. b) The \% of repression of each combination of compounds (over the levels induced by IFN- $\gamma$ was calculated for those genes whose expression was inhibited by one or more antioxidants.

EP2311454 treatment efficiency was evaluated in patients with psoriasis, resulting in significant decrease of BSA, DLQI and PASI levels. This analysis confirmed that EP2311454 is efficacious for the treatment of psoriasis. PASI 75 response rates for individuals treated with EP2311454 is 100\% for severe patients and $80 \%$ for mild to moderate patients. The effect was evident at the earliest assessment (15 days). The results presented here are in line with other studies that suggested that moderate to severe psoriatic plaques should show a therapeutic response to orally administered curcuma [13] or to antioxidant compounds, pointing out that adjuvant therapies are promising solutions for increasing the efficacy of traditional drugs against psoriasis $[14,15]$. In order to understand the cellular mechanisms underlying the efficacy of EP2311454, the effects of individual and combined components within this formula were tested in a model of macrophage inflammatory response in vitro. Analysis of the combined action of indomethacin with other antioxidants has shown that the efficacy of some combinations is greater than the efficacy of individual compounds. The combined use of curcumin and ascorbic acid 6-palmitate resulted in synergistic inhibitory effects on the expression of Cox2, Nos2 and Cxc19, and the presence of indomethacin in the combination further increased repression of Nos2 and Cox2. These results suggest that the combination of curcumin and ascorbic acid 6-palmitate together with indomethacin have increased anti-inflammatory capacity than the antioxidants individually. Together with the clinical results, these data suggest that EP2311454 treatment is a promising strategy to ameliorate the clinical symptoms of psoriasis.

\section{References}

1. Sunil Dogra, Rahul M (2016) Psoriasis: Epidemiology, clinical features, co-morbidities, and clinical scoring. Indian Dermatol Online J 7(6): 471-480.

2. Wisam Alwan, Frank ON (2015) Pathogenesis and Treatment of Psoriasis: Exploiting Pathophysiological Pathways for Precision Medicine. Clin Exp Rheumatol 33(5 Suppl 93): S2-6.

3. Michael Roman, Melvin WC (2017) Spotlight on Brodalumab in the Treatment of Moderate-To-Sever Plaque Psoriasis: Design, Development, and Potential Place in Therapy. Drug Des Devel Ther 11: 2065-2075. 
4. Puig L, Kerkhof, Reich K, Bachelez H, Barker J, et al. (2017) A European Subset Analysis From the Population-Based Multinational Assessment of Psoriasis and Psoriatic Arthritis Shows Country-Specific Features: Results From Psoriasis Patients in Spain. J Eur Acad Dermatol Venereol 31(7): 1176-1182.

5. Lin X, Huang T (2016) Oxidative Stress in Psoriasis and Potential Therapeutic Use of Antioxidants. Free Radic Res 50(6): 585-595.

6. Shuangshuang Z, Xiangdong L, Lihong M, Hongfeng W, Fang F, et al. (2016) Epigallocatechin-3-gallate (EGCG) Inhibits Imiquimod-Induced Psoriasis-Like Inflammation of BALB/c Mice. BMC Complement Altern Med 16(1): 334.

7. Chamcheu JC, Adhami VM, Esnault S, Sechi M, Siddiqui IA, et al. (2017) Dual Inhibition of PI3K/Akt and mTOR by the Dietary Antioxidant, Delphinidin, Ameliorates Psoriatic Features In Vitro and in an Imiquimod-Induced Psoriasis-Like Disease in Mice. Antioxid Redox Signal 26(2): 49-69.

8. Kienhöfer D, Boeltz S, Hoffmann MH (2016) Reactive Oxygen Homeostasis - The Balance for Preventing Autoimmunity. Lupus 25(8): 943-954.

9. Palombo R, Savini I, Avigliano L, Madonna S, Cavani A, (2016) Luteolin7-glucoside Inhibits IL-22/STAT3 Pathway, Reducing Proliferation, Acanthosis, and Inflammation in Keratinocytes and in Mouse Psoriatic Model. Cell Death Dis 7(8): e2344.
10. Antiga E, Bonciolini V, Volpi W, Del Bianco E, Caproni M (2015) Oral Curcumin (Meriva) Is Effective as an Adjuvant Treatment and Is Able to Reduce IL-22 Serum Levels in Patients With Psoriasis Vulgaris. Biomed Res Int 2015: 283634.

11. Zakharova VV, Pletjushkina OY, Zinovkin RA, Popova EN, Chernyak BV (2017) Mitochondria-Targeted Antioxidants and Uncouplers of Oxidative Phosphorylation in Treatment of the Systemic Inflammatory Response Syndrome (SIRS). J Cell Physiol 232(5): 904-912.

12. Xing Lin, Qianshun Chen, Chen Huang, Xunyu Xu (2016) CYLD Promotes TNF- $\alpha$-Induced Cell Necrosis Mediated by RIP-1 in Human Lung Cancer Cells. Mediators Inflamm 2016: 1542786.

13. Gupta SC, Patchva S, Aggarwal BB (2013) Therapeutic Roles of Curcumin: Lessons Learned From Clinical Trials. AAPS J 15(1): 195218.

14. Meng Y, Wang M, Xie X, Di T, Zhao J, et al. (2017) Paeonol Ameliorates Imiquimod-Induced Psoriasis-Like Skin Lesions in BALB/c Mice by Inhibiting the Maturation and Activation of Dendritic Cells. Int J Mol Med 39(5): 1101-1110.

15. Carola X, Joerg JM, Daniel G, Sabine B, Guido S, et al. (2013) Pharmacological Interventions for Pruritus in Adult Palliative Care Patients. Cochrane Database Syst Rev 9(6) :CD008320.

\section{Your next submission with Juniper Publishers will reach you the below assets}

- Quality Editorial service

- Swift Peer Review

- Reprints availability

- E-prints Service

- Manuscript Podcast for convenient understanding

- Global attainment for your research

- Manuscript accessibility in different formats

( Pdf, E-pub, Full Text, Audio)

- Unceasing customer service

Track the below URL for one-step submission https://juniperpublishers.com/online-submission.php 